\title{
BOUNDARY SPECTRA OF UNIFORM FROSTMAN BLASCHKE PRODUCTS
}

\author{
ALEC MATHESON
}

(Communicated by Joseph A. Ball)

\begin{abstract}
A closed set $F$ in the unit circle $\mathbb{T}$ is the boundary spectrum of a uniform Frostman Blaschke product if and only if $F$ is nowhere dense in $\mathbb{T}$.
\end{abstract}

\section{INTRODUCTION}

A Blaschke product $B(z)=z^{m} \prod_{n=1}^{\infty} \frac{\left|a_{n}\right|}{a_{n}} \frac{a_{n}-z}{1-\overline{a_{n}} z}$ is said to satisfy the Frostman condition at the point $\zeta \in \mathbb{T}$ if the quantity

$$
\sigma_{B}(\zeta)=\sum_{n=1}^{\infty} \frac{1-\left|a_{n}\right|}{\left|\zeta-a_{n}\right|}
$$

is finite. Frostman 2, 3] showed that this condition is necessary and sufficient for the Blaschke product $B$ and all of its subproducts to have nontangential limits of modulus one at $\zeta$. The Blaschke product $B$ is said to be uniform Frostman if the quantity

$$
\sigma_{B}=\sup _{\zeta \in \mathbb{T}} \sigma_{B}(\zeta)
$$

is finite. The function $\sigma_{B}(\zeta)$ is the Frostman indicator of $B$ and the number $\sigma_{B}$ is the Frostman constant of $B$. Evidently $\sigma_{B}>1$ for any Blaschke product $B$ with more than one zero. Moreover, any sequence $\left(a_{n}\right)$ satisfying

$$
\sup _{\zeta \in \mathbb{T}} \sum_{n=1}^{\infty} \frac{1-\left|a_{n}\right|}{\left|\zeta-a_{n}\right|}<\infty
$$

is a Blaschke sequence. It was shown by Hruščev and Vinogradov [5] that an inner function is a multiplier of the space of Cauchy transforms if and only if it is a uniform Frostman Blaschke product. An exposition of this result can also be found in 1 .

For any inner function $u$, the boundary spectrum of $u$ is the set

$$
\operatorname{spec}(u)=\left\{\zeta \in \mathbb{T}\left|\liminf _{z \rightarrow \zeta}\right| u(z) \mid=0\right\} .
$$

For a Blaschke product $B, \operatorname{spec}(B)$ is just the accumulation set of the zeros of $B$. In general $u$ admits an analytic continuation across $\mathbb{T} \backslash \operatorname{spec}(u)$. Joseph A. Cima asked whether the boundary spectrum of an inner multiplier of the space of Cauchy

Received by the editors June 30, 2005 and, in revised form, August 10, 2005.

2000 Mathematics Subject Classification. Primary 30D50, 30D40.

(C)2006 American Mathematical Society Reverts to public domain 28 years from publication 
transforms could be the whole unit circle. The theorem below provides a negative answer to this question.

Theorem 1. If $B$ is a uniform Frostman Blaschke product, then the $\operatorname{set} \operatorname{spec}(B)$ is nowhere dense in $\mathbb{T}$.

The next theorem provides a converse to the above theorem.

Theorem 2. If $F$ is a closed nowhere dense subset of $\mathbb{T}$, then there is a uniform Frostman Blaschke product $B$ with $\operatorname{spec}(B)=F$. Moreover, for any $\epsilon>0, B$ can be chosen so that $\sigma_{B}<1+\epsilon$.

Some definitions and known results will be presented in the next section. Theorem 1 will be proved in Section 3, except for a technical lemma, which will be proved in Section 4. Theorem 2 will be proved by an explicit construction in Section 5 .

The author would like to thank Joseph A. Cima and William T. Ross for helpful discussions related to this work, and the referee for suggesting an alternate proof of Theorem 1. This proof will be found in Section 6 .

\section{Definitions AND KNOWn Results}

To each sequence $a=\left(a_{n}\right)$ in the unit disk there is associated a measure $\mu_{a}$ which puts the mass $1-\left|a_{n}\right|^{2}$ at the point $a_{n}$ for each $n$. The sequence $a$ is a Blaschke sequence if $\mu_{a}$ is a finite measure. The (Blaschke) sequence $a$ is a Carleson sequence if $\mu_{a}$ is a Carleson measure, that is, if there is a constant $C$ such that

$$
\mu_{a}\left(Q_{I}\right) \leq C|I|
$$

for every $\operatorname{arc} I$ on $\mathbb{T}$, where $|I|$ denotes the normalized length of $I$, and $Q_{I}$ is the Carleson square

$$
Q_{I}=\{z \in \mathbb{D}|1-| z|<| I \mid \text { and } z /|z| \in I\} .
$$

The sequence $a$ is separated if there is a positive number $\delta$ such that $\rho\left(a_{m}, a_{n}\right)>\delta$ whenever $m \neq n$. Here $\rho(a, b)=\left|\frac{a-b}{1-\bar{a} b}\right|$ is the pseudohyperbolic distance between $a$ and $b$. The sequence $a$ is uniformly separated if there is a positive number $\delta$ such that

$$
\inf _{m} \prod_{n \neq m}\left|\frac{a_{n}-a_{m}}{1-\overline{a_{n}} a_{m}}\right|>\delta .
$$

By Carleson's interpolation theorem, the uniformly separated sequences are precisely the interpolating sequences for $H^{\infty}$. Thus they are often called interpolating sequences, and that practice will be followed here. Clearly, interpolating sequences are separated. It is a result of McKenna 7 that every Carleson sequence can be partitioned into a finite number of interpolating sequences. Conversely, every interpolating sequence is Carleson. All of this and more can be found in Garnett's book [4.

For an angle $\alpha, 0<\alpha<\pi / 2$, and a point $\zeta \in \mathbb{T}$, the Stolz region $S_{\alpha}(\zeta)$ is the convex hull of the closed disk with center 0 and radius $\sin \alpha$ and the point $\zeta$, with the point $\zeta$ removed. Near $\zeta, S_{\alpha}(\zeta)$ is bounded by two straight lines each making angle $\alpha$ with the radius from 0 to $\zeta$.

The next two lemmas are due to Hruščev and Vinogradov [5]. In each case the simple proof is given. If $a$ is a Blaschke sequence, then $\sigma(a)$ is equal to $\sigma_{B}$, where $B$ is the Blaschke product with zero sequence $a$. 
Lemma 1. Let a be a Frostman sequence. Then the number of elements of a in any Stolz region $S_{\alpha}(\zeta)$ does not exceed $2 \sigma(a) /(1-\sin \alpha)$.

Proof. If $z$ is any point in $\mathbb{D}$, the law of cosines gives

$$
|z|^{2}=1+|\zeta-z|^{2}-2|\zeta-z| \cos \theta
$$

where $\theta$ is the angle that the segment from $z$ to $\zeta$ makes with the radius from 0 to $\zeta$. Rearranging this yields

$$
\frac{1-|z|^{2}}{|\zeta-z|}=2 \cos \theta-|\zeta-z|
$$

The chord of the unit circle containing $z$ and $\zeta$ has length $2 \cos \theta$, and so the length of the segment of this chord on the opposite side of $z$ from $\zeta$ has length $2 \cos \theta-|\zeta-z|$. In particular, if $z \in S_{\alpha}(\zeta)$, this length exceeds $1-\sin \alpha$, the length of the segment of the diameter from $-\zeta$ to $\zeta$ lying outside $S_{\alpha}(\zeta)$. If $N$ is the number of elements of the sequence $a$ lying in $S_{\alpha}(\zeta)$, then

$$
N \leq \frac{1}{1-\sin \alpha} \sum_{a_{n} \in S_{\alpha}(\zeta)} \frac{1-\left|a_{n}\right|^{2}}{\left|\zeta-a_{n}\right|} \leq \frac{2 \sigma(a)}{1-\sin \alpha}
$$

Lemma 2. Let a be a Frostman sequence. Then $\mu_{a}$ is a Carleson measure.

Proof. Let $Q$ be a Carleson square. Then

$$
\begin{aligned}
\mu_{a}(Q) & =\sum_{a_{n} \in Q} 1-\left|a_{n}\right|^{2} \\
& \leq c|I| \sum_{a_{n} \in Q} \frac{1-\left|a_{n}\right|^{2}}{\left|\zeta-a_{n}\right|} \\
& \leq 2 c|I| \sigma(a),
\end{aligned}
$$

where $c$ is an absolute constant, $|I|$ is the length of the arc $I$ upon which $Q$ rests, and $\zeta$ is the midpoint of $I$.

Lemma 3. Let $B$ be a Blaschke product admitting a factorization $B=B_{1} B_{2} \ldots B_{n}$. Then $\operatorname{spec}(B)=\bigcup_{k=1}^{n} \operatorname{spec}\left(B_{k}\right)$.

Proof. This is obvious.

\section{Proof of Theorem 1}

The proof of Theorem 1 depends on the following lemma. This lemma will be proved in Section 4

Lemma 4. Let $F$ be a countable subset of $\mathbb{D}$ such that for some fixed $\alpha, 0<\alpha<\frac{\pi}{2}$, there is an integer $N$ such that no Stolz region $S_{\alpha}(\zeta)$ contains more than $N$ points from $F$. Then $F$ admits a partition $F=\bigcup_{k=0}^{2 N-1} F_{k}$, where $F_{0}=F \cap \overline{D(0, \sin \alpha)}$ and each $F_{k}, k=1, \ldots, 2 N-1$, has at most one point in each Stolz region $S_{\alpha}(\zeta)$.

If $F \subset \mathbb{T}$ is a finite union $F=\bigcup_{k=1}^{n} F_{k}$ of closed sets $F_{k}$ nowhere dense in $\mathbb{T}$, then it is clear that $F$ is also a closed set nowhere dense in $\mathbb{T}$. If $B$ is a uniform Frostman Blaschke product which can be written as a finite product $B=\prod_{k=1}^{n} B_{k}$ of Blaschke products, then each factor $B_{k}$ is uniform Frostman, and, by Lemma 3 , 
it will be enough to show that each factor $B_{k}$ satisfies the conclusion of Theorem 1 . Because the zero sequence of $B$ is Carleson, $B$ can be factored as a finite product so that each factor $B_{k}$ is interpolating, and, in particular, has simple zeros. Then an application of Lemma 4 shows that $B$ admits a factorization $B=\prod_{k=1}^{n} B_{k}$, where each $B_{k}$ has simple zeros and has the property that no Stolz region $S_{\alpha}(\zeta)$ contains more than one zero of $B_{k}$.

Now suppose that $B$ is a Blaschke product with the property that every Stolz region $S_{\alpha}(\zeta)$ contains at most one zero of $B$. Let $F=\operatorname{spec}(B)$, and let $\zeta$ be an interior point of $F$. There is a positive number $\epsilon$ such that $F$ contains the arc $I_{\zeta}=\mathbb{T} \cap D(\zeta, \epsilon)$. For each $z \in \mathbb{D}$, let

$$
J_{z}=\left\{\xi \in \mathbb{T}: z \in S_{\alpha}(\xi)\right\} .
$$

It is easy to see that there is a positive number $\eta<\epsilon$ which depends only on $\epsilon$ and $\alpha$ such that $J_{z} \subset I_{\zeta}$ for every $z \in \mathbb{D} \cap D(\zeta, \eta)$. Since $\zeta \in \operatorname{spec}(B), B$ has a zero $z$ which lies in the neighborhood $\mathbb{D} \cap D(\zeta, \eta)$ of $\zeta$. Because each Stolz region contains at most one zero of $B$, the interior of the convex hull of $z$ and $J_{z}$ can contain no zeros of $B$, and so the interior of $J_{z}$ is disjoint from $\operatorname{spec}(B)$. This is a contradiction.

\section{Proof of Lemma 4}

Suppose that $F$ is a set satisfying the hypotheses of Lemma 4, that is, there is an $\alpha, 0<\alpha<\frac{\pi}{2}$, and a positive integer $N$ such that each Stolz region $S_{\alpha}(\zeta)$ contains at most $N$ points from $F$. Let $F_{0}=F \cap \overline{D(0, \sin \alpha)}$, and let $F^{\prime}=F \backslash F_{0}$. Since $F^{\prime}$ is countable, its elements can be listed in a sequence $\left(a_{n}\right)$, arranged so that the moduli of the elements form an increasing sequence. For each positive integer $n$, let $I_{n}=J_{a_{n}}$ be the arc of $\mathbb{T}$ such that $a_{n} \in S_{\alpha}(\zeta)$ for each $\zeta \in I_{n}$. Clearly, $\left|I_{1}\right| \geq\left|I_{2}\right| \geq \cdots$. The sequence of intervals $\left(I_{n}\right)$ has the property that no point of the unit circle is contained in more than $N$ of the $I_{n}$. This follows immediately from the observation that $\zeta \in I_{n}$ if and only if $a_{n} \in S_{\alpha}(\zeta)$.

Lemma 5. The sequence $\mathbb{N}$ of natural numbers can be partitioned into at most $2 N-1$ subsequences $M_{k}$ such that for each $k$, the sequence of intervals $\left\{I_{n}: n \in\right.$ $\left.M_{k}\right\}$ is pairwise disjoint.

Proof. The proof mimics the proof of Besicovitch's covering theorem [6. pp. 30-34]. In order to construct $M_{1}$, let $n_{1}=1$, and let $n_{2}$ be the smallest positive integer such that $I_{n_{1}}$ and $I_{n_{2}}$ are disjoint. If $n_{1}<n_{2}<\cdots<n_{l}$ have been selected so that the intervals $I_{n_{i}}, 1 \leq i \leq l$, are pairwise disjoint, let $n_{l+1}$ be the smallest integer greater than $n_{l}$ such that $I_{n_{l+1}}$ is disjoint from the intervals $I_{n_{i}}, 1 \leq i \leq l$. Continuing this process as long as possible creates $M_{1}$.

Next, $M_{2}$ is constructed from $\mathbb{N} \backslash M_{1}$ in the same way. First, let $n_{1}$ be the smallest positive integer in $\mathbb{N} \backslash M_{1}$. Next let $n_{2}$ be the smallest positive integer in $\mathbb{N} \backslash M_{1}$ such that $I_{n_{2}}$ is disjoint from $I_{n_{1}}$. The process continues as above.

As long as $\bigcup_{k=1}^{l} M_{i} \neq \mathbb{N}$ this process can be continued to create $M_{l+1}$. If $\bigcup_{k=1}^{K} M_{k}=\mathbb{N}$ for some $K \leq 2 N-1$, then the lemma is proved. Otherwise there is a smallest positive integer $n$ not belonging to $\bigcup_{k=1}^{2 N-1} M_{k}$. Then by construction, there is, for each $k, 1 \leq k \leq 2 N-1$, an integer $n_{i} \in M_{k}$ such that $n_{i}<n$ and $I_{n_{i}}$ intersects $I_{n}$, since otherwise, $n$ would have been selected as a member of $M_{k}$. Since $I_{n}$ is no longer than $I_{n_{k}}$ for each $k, I_{n_{k}}$ must contain at least one of the 
endpoints of $I_{n}$. Consequently, at least one of the endpoints of $I_{n}$ belongs to at least $N$ intervals, contradicting the hypotheses.

Applying the lemma, there is a partition $\mathbb{N}=\bigcup_{k=1}^{K} M_{k}$ of $\mathbb{N}$ with $K \leq 2 N-1$, such that for each $k, 1 \leq k \leq K$, the sequence $\left(I_{n}\right)_{n \in M_{k}}$ is pairwise disjoint. For each $k$, let $F_{k}=\left\{a_{n}: n \in M_{k}\right\}$. Evidently $F_{k}$ has the property that it intersects any Stolz region in at most one point. This completes the proof of Lemma 4 .

\section{A Construction}

Suppose that $F$ is a closed subset of $\mathbb{T}$ with no interior, and let $\left(I_{n}\right)$ be the sequence of arcs contiguous to $F$, that is,

$$
\mathbb{T} \backslash F=\bigcup_{n} I_{n},
$$

and the $I_{n}$ are pairwise disjoint open arcs in $\mathbb{T}$. Let $a_{n}, b_{n}$ denote the endpoints of $I_{n}$, with $a_{n}$ coming before $b_{n}$ as the arc $I_{n}$ is traversed in the counterclockwise direction. Because $F$ is nowhere dense in $\mathbb{T}$, it is the closure of the set of endpoints of the $I_{n}$.

The proof of Theorem 2 proceeds by constructing, for each $I_{n}$, a Blaschke product $B_{n}$ whose zeros lie over $I_{n}$ and accumulate at the two endpoints $a_{n}$ and $b_{n}$, and so that the Frostman indicator $\sigma_{B_{n}}(\zeta)$ is small for $\zeta \notin I_{n}$, and not too big for $\zeta \in I_{n}$. Specifically, let $\epsilon>0$, and let $\epsilon_{n}$ be such that $\sum_{n} \epsilon_{n}<\epsilon / 2$. Suppose that for each $n$ a Blaschke product $B_{n}$ has been constructed such that the zeros of $B_{n}$ lie over $I_{n}$ and accumulate at both endpoints of $I_{n}$ and such that

$$
\sigma_{B_{n}}(\zeta)< \begin{cases}1+\epsilon / 2 & \text { if } \zeta \in I_{n} \\ \epsilon_{n} & \text { if } \zeta \notin I_{n} .\end{cases}
$$

Now let $B=\prod_{n} B_{n}$. Since the Frostman indicator $\sigma_{B}(\zeta)$ is clearly additive over products, it follows that this product converges uniformly on compact sets to a Blaschke product $B$, and that $\sigma_{B}<1+\epsilon$. Obviously, $\operatorname{spec}(B)=F$. Up to the construction of $B_{n}$, that completes the proof of Theorem 2 .

In order to construct the $B_{n}$, it will be enough to consider a single arc $I$, and construct, for a given constant $\eta, 0<\eta<1$, a Blaschke product $B$ whose zeros lie over $I$ and accumulate at one of the endpoints of $I$ and such that

$$
\sigma_{B}(\zeta)< \begin{cases}1+\eta & \text { if } \zeta \in I^{\prime} \\ \eta & \text { otherwise }\end{cases}
$$

where $I^{\prime}$ is a subarc of $I$ sharing the endpoint in question and having length less than or equal to one half of the length of $I$. If $B_{1}$ and $B_{2}$ are the Blaschke products constructed for each of the endpoints of $I$, then clearly the product $B=B_{1} B_{2}$ satisfies

$$
\sigma_{B}(\zeta)<\left\{\begin{array}{lc}
1+2 \eta & \text { if } \zeta \in I, \\
2 \eta & \text { otherwise }
\end{array}\right.
$$

Now suppose that 1 is one of the endpoints of $I$ and $\alpha, 0<\alpha<\pi / 4$, is such that $I$ contains the shorter interval $I^{\prime \prime}$ with endpoints 1 and $e^{i \alpha}$, and finally that $I^{\prime \prime}$ has length less than or equal to one fourth of the length of $I$. Let $I^{\prime}$ be the arc containing $I^{\prime \prime}$ with endpoints 1 and $e^{2 i \alpha}$. Now, for $n=0,1, \ldots$, let $a_{n}=r_{n} e^{i \theta_{n}}$, 
where $1-r_{n}=\frac{\eta}{4} \frac{\alpha}{4^{n}}$ and $\theta_{n}=\frac{\alpha}{2^{n}}$. Because of the choice of $\alpha$, for each $n$ and any $t$

$$
\left|e^{i t}-r_{n}\right|^{2}>\left(1-r_{n}\right)^{2}+t^{2}
$$

so that in any expression the denominator $\left|e^{i \theta}-a_{n}\right|$ can be replaced by $1-r_{n}$ or by $\left|\theta-\theta_{n}\right|$ as appropriate. Let

$$
\sigma(\theta)=\sum_{n=0}^{\infty} \frac{1-r_{n}}{\mid e^{i \theta}-r_{n} e^{i \theta_{n} \mid}}
$$

First suppose that $2 \theta_{0} \leq \theta \leq \pi$. Then for each $n,\left|\theta-\theta_{n}\right| \geq\left|\theta_{0}\right|=\alpha$, so that

$$
\sigma(\theta) \leq \frac{1}{\alpha} \sum_{n=0}^{\infty}\left(1-r_{n}\right) \leq \frac{\eta}{4} \sum_{n=0}^{\infty} \frac{1}{4^{n}}=\frac{4}{3} \frac{\eta}{4}=\frac{\eta}{3}<\eta .
$$

Next, if $\theta_{0} \leq \theta \leq 2 \theta_{0}$, then for $n \geq 1,\left|\theta-\theta_{n}\right| \geq\left|\theta_{0}-\theta_{1}\right|=\frac{\alpha}{2}$, so that

$$
\begin{aligned}
\sigma(\theta) & \leq \frac{1-r_{0}}{\left|e^{i \theta}-a_{n}\right|}+\sum_{n=1}^{\infty} \frac{1-r_{n}}{\left|e^{i \theta}-a_{n}\right|} \\
& \leq 1+\frac{2}{\alpha} \sum_{n=1}^{\infty} \frac{\eta}{4} \frac{\alpha}{4^{n}} \\
& =1+\frac{2}{3} \frac{\eta}{4}=1+\frac{\eta}{6}<1+\eta .
\end{aligned}
$$

Now suppose that $\theta_{n} \leq \theta \leq \theta_{n-1}$. Suppose that $\theta$ is closer to $\theta_{n}$ than to $\theta_{n-1}$, i.e., $\theta_{n} \leq \theta \leq \frac{\theta_{n}+\theta_{n-1}}{2}$. If $k<n-1$, then $\theta_{k}-\theta \geq \theta_{k}-\theta_{k+1}=\frac{\alpha}{2^{k}}-\frac{\alpha}{2^{k+1}}=\frac{\alpha}{2^{k+1}}$. Similarly, if $k>n, \theta-\theta_{k} \geq \theta_{k-1}-\theta_{k}=\frac{\alpha}{2^{k}} \geq \frac{\alpha}{2^{k+1}}$. If $k=n-1$, then $\theta_{n-1}-\theta \geq \theta_{n-1}-\frac{\theta_{n}+\theta_{n-1}}{2}=\frac{\theta_{n-1}-\theta_{n}}{2}=\frac{\alpha}{2^{n+1}}$. Thus

$$
\begin{aligned}
\sigma(\theta) & =\sum_{k=0}^{n-1} \frac{1-r_{k}}{\left|e^{i \theta}-a_{k}\right|}+\frac{1-r_{n}}{\left|e^{i \theta}-a_{n}\right|}+\sum_{k=n+1}^{\infty} \frac{1-r_{k}}{\left|e^{i \theta}-a_{k}\right|} \\
& \leq \frac{\eta}{4} \sum_{k=0}^{n-1} \frac{2^{n+1}}{4^{n}}+1+\frac{\eta}{4} \sum_{k=n+1}^{\infty} \frac{2^{n+1}}{4^{n}} \\
& <1+\frac{\eta}{2} \sum_{n=0}^{\infty} \frac{1}{2^{n}} \\
& =1+\eta .
\end{aligned}
$$

A similar argument works if $\theta$ is closer to $\theta_{n-1}$ than it is to $\theta_{n}$. Finally, if $-\pi \leq$ $\theta \leq 0$, then $\theta_{n}-\theta \geq \theta_{n}$, and so

$$
\begin{aligned}
\sigma(\theta) & \leq \sum_{n=0}^{\infty} \frac{1-r_{n}}{\theta_{n}} \\
& =\frac{\eta}{4} \sum_{n=0}^{\infty} \frac{2^{n}}{4^{n}} \\
& =\frac{\eta}{2}<\eta .
\end{aligned}
$$


This completes the construction of $B$ and the proof of Theorem 2 .

\section{Conclusion}

Finally, the referee has suggested an alternate, less quantitative, proof of Theorem 1 which uses the Baire category theorem. This proof proceeds as follows. Let $S_{\alpha}^{\circ}(\zeta)$ be the interior of $S_{\alpha}(\zeta)$ As observed by Frostman, each $S_{\alpha}^{\circ}(\zeta)$ contains only finitely many zeros of $B$, else $B$ would not have a radial limit of modulus one at $\zeta$. Now suppose $I$ is a closed arc in the boundary spectrum $F$ of $B$. For each positive integer $n$, let

$$
E_{n}=\left\{\zeta \in I: S_{\alpha}^{\circ}(\zeta) \text { contains no zero } z \text { of } B \text { with }|z| \geq 1-\frac{1}{n}\right\} .
$$

Clearly $\bigcup_{n=1}^{\infty} E_{n}=I$. Since each $S_{\alpha}^{\circ}(\zeta)$ is open, each $E_{n}$ is closed. By Baire's theorem some $E_{n}$ contains an open arc $J$. Evidently $J$ is disjoint from $F$, and that is a contradiction.

\section{REFERENCES}

1. Joseph A. Cima, Alec L. Matheson, and William T. Ross, The Cauchy transform, Mathematical Surveys and Monographs, vol. 125, American Mathematical Society, Providence, RI, 2006.

2. E. F. Collingwood and A. J. Lohwater, The theory of cluster sets, Cambridge Tracts in Mathematics and Mathematical Physics, No. 56, Cambridge University Press, Cambridge, 1966. MR0231999(38:325)

3. Otto Frostman, Sur les produits de Blaschke, Kungl. Fysiografiska Sällskapets i Lund Förhandlingar [Proc. Roy. Physiog. Soc. Lund] 12 (1942), no. 15, 169-182. MR0012127|(6:262e)

4. John B. Garnett, Bounded analytic functions, Pure and Applied Mathematics, vol. 96, Academic Press Inc. [Harcourt Brace Jovanovich Publishers], New York, 1981. MR628971 (83g:30037)

5. S. V. Hruščev and S. A. Vinogradov, Inner functions and multipliers of Cauchy type integrals, Ark. Mat. 19 (1981), no. 1, 23-42. MR625535 (83c:30027)

6. Pertti Mattila, Geometry of sets and measures in Euclidean spaces, Cambridge Studies in Advanced Mathematics, vol. 44, Cambridge University Press, Cambridge, 1995. MR 1333890 (96h:28006)

7. P. J. McKenna, Discrete Carleson measures and some interpolation problems, Michigan Math. J. 24 (1977), no. 3, 311-319. MR0481016 (58:1163)

Department of Mathematics, Lamar University, P.O. Box 10047, Beaumont, Texas 77710

E-mail address: matheson@math.lamar.edu 